\title{
Inference of Molecular Subtypes of Uterine Corpus Endometrioid Carcinoma with Different Survival Based on Cancer Hallmarks-associated Long Non- Coding RNAs and Gene Modules
}

Nan Lv ( $\nabla 13936538221 @ 163 . c o m)$

Second Affiliated Hospital of Harbin Medical University

Shuyu Zhao

Department of Endocrinology, Second Affiliated Hospital of Harbin Medical University

Yan Li

Department of Endocrinology, Second Affiliated Hospital of Harbin Medical University

Tianyi Liu

Department of Endocrinology, Second Affiliated Hospital of Harbin Medical University

Xiaodan Chu

Department of Endocrinology, Second Affiliated Hospital of Harbin Medical University

\section{Research}

Keywords: Long non-coding RNA, cancer hallmark, uterine corpus endometrioid carcinoma, survival, molecular subtypes

Posted Date: June 30th, 2020

DOI: https://doi.org/10.21203/rs.3.rs-37407/v1

License: (c) (i) This work is licensed under a Creative Commons Attribution 4.0 International License.

Read Full License 


\section{Abstract}

Background: Uterine corpus endometrioid carcinoma (UCEC), a common gynecological malignancy with high incidence, affects the mental and physical health of women. It is increasingly evident that long noncoding RNAs (IncRNAs) have causative roles in cancers including UCEC. However, very little is known about the cancer hallmark-related risks for UCEC based on IncRNAs and genes.

Methods: In this study, a computational integrated pipeline was development to evaluate cancer hallmark-related risk for each UCEC patient based on gene and IncRNA expression.

Results: Some UCEC-specific cancer hallmark-related genes and IncRNAs were identified. Core modules were extracted from co-expressed UCEC-specific IncRNAs and genes networks for each cancer hallmark. Some core modules showed specific features and functions. Follow a multi-dimensional rank approach and core modules, each UCEC sample was given an integrated cancer hallmark-related risk score. We divided all the UCEC patients to diverse hallmark risk groups including multi-hallmark, media-hallmark and single-hallmark groups and they showed different prognosis. We also identified some key IncRNAs which participated in multiple kinds of cancer hallmarks. These key IncRNAs were associated with some essential pathways such as estrogen receptor signaling.

Conclusions: In conclusion, the present study provide novel insights to the classification and treatment of UCEC.

\section{Background}

Uterine corpus endometrioid carcinoma (UCEC) is the sixth-most-common leading cause of cancer-related death among women in the United States[1,2], with an estimated 65,620 new cases and 12,590 deaths in 2020 [3]. Over time, the number of newly diagnosed UCEC in the United States has steadily increased and this trend is expected to continue [4]. Most UCEC women of early stages diagnosed show favorable outcomes. However, there are some low-grade, early, well differentiated UCEC women in which unexpected recurrence and adverse outcomes may occur. UCEC patients usually have diverse survival and drug response. Thus, UCEC is one of the few human malignant tumors for which mortality is increasing, which underscores the urgent need to construct novel and effective strategies for distinguishing subtypes with diverse survival and drug response of UCEC.

Recent years, many kinds of non-coding RNAs have been discovered and researched in multiple kinds of diseases including cancer [5, 6]. Long non-coding RNA (IncRNA) was a major kind of non-coding RNAs which $>200 \mathrm{nt}$ in length $[7,8]$. Accumulating evidence has suggested that IncRNAs serve as important and essential roles in physiological and pathological processes of various cancers $[9,10]$. Numerous studies have reported that the occurrence, development, prognosis and treatment of UCEC were closely related to the abnormal expression of a large number of IncRNAs. For example, IncRNA SNHG16 induced by TFAP2A modulates glycolysis and proliferation of UCEC and is associated with poor survival rate [11]. Yao et al. reported that IncRNA LSINCT5 is up-regulated and significantly inhibited cell proliferation, cell 
cycle progression, and induced apoptosis in UCEC. Thus, comprehensive and systematic exploration of the characteristics about IncRNA in UCEC could provide assistance for identifying novel molecular associations of potential mechanistic significance in the development of UCEC.

It is generally known that the biology of cancer is extremely complex, individualized and various. However, some key traits have been revealed to reduce cancer complexity during the past decade. These traits could be represented by a few distinctive and complementary capabilities (which are considered as "cancer hallmarks") that could promote tumor growth and metastasis. These cancer hallmarks could provide a logical framework for understanding the significant diversity of multiple kinds of cancers [12]. Hanahan and Weinberg proposed six hallmarks and ten hallmarks of cancer in 2000 and 2011 [13, 14]. These two researches both suggest that multiple kinds of cancers share some common cancer hallmarks which dominate the convert from normal cells to cancer cells. These ten cancer hallmarks are effective principles to depict the characteristics of cancers. However, we have little insight into how to use these hallmarks to depict the roles of IncRNAs and distinguish UCEC patients.

In present study, we developed an integrated algorithm for evaluating hallmark-related risks of UCEC patients and distinguishing them to diverse groups based on gene and IncRNA expression profiles (Figure 1). Some UCEC-specific IncRNAs and genes in ten kinds of hallmarks were identified. Co-expressed UCEC-specific IncRNAs and genes networks in diverse cancer hallmark were constructed. Some core modules were extracted from these co-expressed UCEC-specific IncRNAs and genes networks. Each UCEC patient was given a comprehensive score for a specific core module in each cancer hallmark based on multi-dimensional rank approach. All the UCEC patients were divided to diverse hallmark risk groups including multi-hallmark, media-hallmark and single-hallmark groups. These diverse hallmark risk groups were associated with different survival. Some key IncRNAs which were related to multiple cancer hallmarks were also discovered and showed specific functions. Collectively, this study clarified the roles of cancer hallmark-related IncRNAs in UCSC and distinguished them to diverse risk groups.

\section{Methods}

\section{Collection of gene and IncRNA expression profiles of UCEC patients}

The IncRNA expression and gene expression (level 3) data, as well as clinical data of UCEC patients were obtained from The Cancer Genome Atlas (TCGA, Release: 2019-07-21). The download link of these data was https://gdc.xenahubs.net/download/TCGA-UCEC.htseq_fpkm.tsv.gz. We also obtained genome annotation data, including genome sites and symbols of genes and IncRNAs from GENCODE 31 (19.06.19). The dataset includes 545 UCEC tumor and 38 control samples. All the IncRNAs and genes which were not expressed in all samples would be excluded. The minimum value of all samples were given to any remaining expression values of 0 . All the expression values were transformed follow $\log 2($ value+1).

\section{Gathering cancer hallmark-related $\mathrm{GO}$ terms and genes}


All the cancer hallmark-related GO terms were obtained from a previous study [15]. We downloaded all the genes of these cancer hallmark-related GO terms from Gene Ontology using AmiGo (version: 2.5.12; http://amigo.geneontology.org/amigo) [16]. Thus, all the cancer hallmark-related genes were got for follow analysis.

\section{Identification of UCEC-specific cancer hallmark-related genes and IncRNAs}

- test was applied to identify differential expressed cancer hallmark-related genes and IncRNAs between UCEC and control normal samples based on expression profiles. IncRNAs and cancer hallmark-related genes were considered as UCEC-specific IncRNAs and cancer hallmark-related genes if they were differentially expressed $(P<0.01)$. All the UCEC-specific IncRNAs and cancer hallmarkrelated genes were divided to up- and down-regulated IncRNAs and genes based on fold values.

\section{Construction of UCEC-specific cancer hallmark-related genes and IncRNAs co-expressed networks and identification of core modules}

We calculated Pearson's correlation coefficients (PCCs) for each UCEC-specific cancer hallmark-related gene and IncRNA pair in all cancer hallmarks. The UCEC-specific gene and IncRNA pairs which their PCCs were higher than 0.3 or smaller than -0.3 and the $p$-values were smaller than 0.01 were extracted and considered as co-expressed UCEC-specific gene and IncRNA pairs. UCEC-specific cancer hallmark-related genes and IncRNAs co-expressed networks were constructed by Cytoscape 3.3.0 (http://www.cytoscape.org/) based on co-expressed pairs which their PCCs were higher than 0.5 or smaller than -0.5. In order to more accurately identify cancer hallmark-related genes and IncRNAs pairs in UCEC, we extracted core modules from co-expressed networks using MCODE module in cytoscape. Thus, certain numbers of core modules were identified for each cancer hallmark in UCEC.

\section{Evaluating hallmark-related risk for UCEC patients and dividing them to diverse risk groups based on core modules}

For each UCEC patient, risk score in each cancer hallmark was calculated and given. Multidimensional rank approach was applied to obtain risk score for each cancer hallmark based on genes and IncRNAs expression values in core modules. Up- and down-regulated genes and IncRNAs ranked in positive and negative order. 1000 permutation test was performed follow randomly perturbing cancer samples. The UCEC patient was considered as a risk sample for a specific cancer hallmark if permutation $P$ values was smaller than 0.05 in any core module. Then, all the UCEC patients were divided to non-hallmark (0 risk hallmark), media-hallmark (1-5 risk hallmarks) and multi-hallmark groups (6-8 risk hallmarks).

\section{Survival analysis for diverse risk cancer hallmark groups and functional analysis for UCEC-specific genes and IncRNAs}

Kaplan-Meier survival analysis was performed for diverse risk cancer hallmark groups. Log-rank test was using to asses statistical significance $(P<0.05)$. R 3.6.2 framework was performed for all analyses. Enrichr tools (http://amp.pharm.mssm.edu/Enrichr) with default parameters was used for functional 
analysis based on UCEC-specific genes in each cancer hallmark [17]. Significantly enriched pathways ( $\mathrm{P}<$ 0.05) were obtained for each cancer hallmark in UCEC.

\section{Results}

\section{Some IncRNAs and cancer hallmark-related genes were specific and co-expressed in UCEC}

In order to depict the roles of cancer hallmark in UCEC, we identified UCEC-specific IncRNAs and cancer hallmark-related genes by differential expression. 1880 (12.43\%) UCEC-specific IncRNAs were identified between UCEC and control samples (Figure 2A). These UCEC-specific IncRNAs included 891 and 989 upand down-regulated IncRNAs (Figure 2B). UCEC-specific genes were also identified for each cancer hallmark (Figure 2C). In all kinds of cancer hallmarks, there were more than $50 \%$ UCEC-specific genes. Specially, there was $70.57 \%$ UCEC-specific genes in cancer hallmark genome instability and mutation. The results indicated that these cancer hallmark-related genes maybe serve as essential roles in UCEC. We assumed that IncRNAs and cancer hallmark-related genes could function by cooperating in UCEC. Thus, some co-expressed IncRNAs and cancer hallmark-related genes pairs were identified in UCEC for each kind of cancer hallmark. Most PCCs of co-expressed IncRNAs and cancer hallmark-related genes pairs were concentrated between 0.3 and 0.5 (Figure 2D). There were 8631 co-expressed IncRNAs and cancer hallmark-related genes pairs which their PCCs were higher than 0.5 (Figure 2E). In each kind of cancer hallmark, the numbers of pairs, IncRNAs and genes were diverse (Figure 2F). For example, there were 2525, 365 and 384 pairs, genes and IncRNAs in cancer hallmark self sufficiency in growth signals. However, only there were 84, 47 and 12 pairs, genes and IncRNAs in cancer hallmark reprogramming energy metabolism. The results indicated that diverse cancer hallmarks play different roles in UCEC. All above results indicated that IncRNAs and cancer hallmark-related genes cooperative pairs were important in UCEC.

\section{Some core modules were extracted from co-expressed IncRNAs and cancer hallmark-related genes networks in each cancer hallmark}

For each cancer hallmark, co-expressed IncRNAs and cancer hallmark-related genes which their PCCs were higher than 0.5 were extracted for constructing co-expressed networks. In cancer hallmark evading apoptosis, IncRNAs and cancer hallmark-related genes co-expressed network was constructed (Figure $3 A$ ). This co-expressed network contained 380 nodes (234 UCEC-specific IncRNAs and 147 cancer hallmark-related genes) and 906 edges. We found that some cancer hallmark-related genes and IncRNAs such as SLC25A27, AC005288 and GD5-AS1 played core roles in this co-expressed network. Most of cancer hallmark-related genes and IncRNAs showed positive correlations in UCEC. In eight cancer hallmarks, there were diverse numbers of core modules were identified (Figure 3B). Cancer hallmarks insensitivity to antigrowth signals, self sufficiency in growth signals and tissue invasion and metastasis had most core modules. The numbers of cancer hallmark-related genes and IncRNAs were also different (Figure $3 C$ ). For example, there were more IncRNA in core module 1 in cancer hallmark insensitivity to antigrowth signals. These core modules maybe show specific functions. For example, a core module in 
cancer hallmark evading apoptosis contained three IncRNAs and three genes (Figure 3D). PSMB8, PSMB10, PSME2 and PSMB8-AS1 were all proteasome-related genes or IncRNAs. The proteasome is a multicatalytic proteinase complex which is characterized by its ability to cleave peptides. Specially, cancer hallmark-related gene PSMB8 and IncRNA PSMB8-AS1 showed strong positive correlation $(\mathrm{P}<$ $0.001, P C C=0.79$ ). These genes and IncRNAs in this core module showed close interactions. Another core module also showed close interactions (Figure 3E). All the results explained these core modules in coexpressed networks could function and serve as specific biomarks in UCEC.

\section{Specific cancer hallmark-related risk were evaluated for each UCEC patient based on core modules}

We inferred that each UCEC patient maybe have diverse hallmark-related risk. Thus, we calculated risk scores for each UCEC patient based on core modules in each cancer hallmark. Only eight cancer hallmarks were extracted for calculating risk scores due to core modules. The density distribution of risk scores in all core modules of each cancer hallmark were similar (Figure 4A). Only a small number of UCEC patients showed higher risk scores. The differences of average risk scores for diverse top 20 core modules were also present (Figure 4B). These 20 core modules were significantly associated with more UCEC patients (Figure 4C). For example, there were $32.03 \%$ samples were significant in core module 1 in cancer hallmark insensitivity to antigrowth signals. This core module was a key module which had most related samples and also been explained in above results. We also discovered the percent of significant risk-related samples in 0\%corresponding top ranked samples. 70\% UCEC samples ranked before corresponding orders in most core modules (Figure 4D). These results indicated that UCEC patients showed differences of cancer hallmark-related risk scores.

\section{UCEC groups with diverse cancer hallmark risk showed specific features}

Each UCEC patient could be associated with diverse cancer hallmark follow above pipeline. The numbers of UCEC in each cancer hallmark were different (Figure 5A). For example, there were more than 350 UCEC samples were associated with cancer hallmark tissue invasion and metastasis. The cancer hallmark reprogramming energy metabolism was related to 300 UCEC samples. Specially, some UCEC patients were associated with multiple cancer hallmarks. 13.59\% UCEC patients had some relationships with any kinds of cancer hallmarks (Figure 5B). 11.55\% UCEC patients were only related to one kind of cancer hallmark. Thus, we could divide all the UCEC patients to diverse groups with different numbers of cancer hallmarks (Figure 5C). The three diverse groups contained non-, media- and multi-hallmarks. $70.76 \%$ samples belonged to media-hallmarks groups. The UCEC patients with more cancer hallmarks usually had better survival days (Figure 5D). In addition, we also divided all the UCEC patients to another three cancer hallmark-related risk groups based on hierarchical clustering (Figure 5E). We also discovered that these diverse cancer hallmark-related risk groups showed different prognosis (Figure 5F, G). Group 2 significantly had better survival than group 1 and $3(P=0.014$ and 0.023$)$. All the results suggested that diverse hallmark-related risk groups had respective features and prognosis.

Some key IncRNAs could participate in multiple kinds of cancer hallmarks and showed specific functions 
In order to further depict the roles of IncRNAs in cancer hallmarks for UCEC, we extracted some key IncRNAs which participate in multiple kinds of cancer hallmarks. There were 11 IncRNAs were associated with more than three kinds of cancer hallmarks (Figure 6A). IncRNAs AL590764.1, ANKRD10-IT1, NORD and AP000766.1 could participated in four kinds of cancer hallmarks (Figure 6B). However, the classes of these four kinds of cancer hallmarks were diverse. We inferred that these IncRNAs may serve as essential roles in UCEC. Thus, we further performed functional analysis for these IncRNAs in each kind of cancer hallmark. The IncRNAs were enrichment in some essential functions for cancer development (Figure 6C). For example, IncRNAs associated with evading apoptosis were enrichment in some hormone-related pathways such as negative regulation of intracellular estrogen receptor signaling, negative regulation of intracellular steroid hormone receptor signaling and regulation of intracellular estrogen receptor signaling. The estrogen receptor status is reported to be an important marker of UCEC $[18,19]$. In addition, we found most IncRNAs were associated with gap junction assembly pathway. Nishimura $\mathrm{M}$ et al. reported that gap junctional intercellular communication was suppressed via $5^{\prime} \mathrm{CpG}$ island methylation in promoter region of E-cadherin gene in UCEC cells [20]. These results indicated that cancer hallmarkrelated IncRNAs could serve as essential roles in UCEC.

\section{Discussion}

In this study, a calculated integrated approach which evaluate cancer hallmark-risk for each UCEC patient based on gene and IncRNA expression was developed. Some core modules were extracted from coexpressed UCEC-specific IncRNAs and genes networks in diverse cancer hallmark. All the UCEC patients were divided to diverse hallmark risk groups based on respective comprehensive risk scores. These diverse hallmark risk groups showed different survival. Some key IncRNAs were related to multiple cancer hallmarks and showed specific functions.

Dividing cancer samples to groups with diverse molecular characteristics could provide assistance for cancer diagnosis and individualized treatment. Wright GW et al. described an algorithm that determines the probability that a patient's lymphoma belongs to one of seven genetic subtypes based on its genetic features [21]. Lee $S$ et al. presented an explainable deep learning model with attention mechanism and network propagation for cancer subtype classification [22]. Most of these developed clarification methods only focused on coding genes. In our work, IncRNAs were considered as essential and key factors for constructing the computational approach about cancer patients classification. The computational approach not only divided UCEC patients to diverse hallmark risk groups but also discovered some key IncRNAs which serve as important roles in UCEC. These IncRNAs were significantly enriched in some essential cancer development functions.

Analysis of cancer hallmarks could greatly improve our understanding of the occurrence, development and metastasis of many cancer types. In present study, we applied cancer hallmarks to evaluate and distinguish UCEC patients. We discovered that most cancer hallmark-related genes were differentially expressed in UCEC. It indicated that cancer hallmark-related genes maybe had essential functions in UCEC. Specially, we found that each UCEC patient showed obvious differences for risk of cancer 
hallmarks. Although cancer hallmarks were considered as common properties for cancer, each UCEC patient still showed personalized features. This result supported that there were great differences among different cancer patients and the application of personalized medicine in cancer treatment is very important. Thus, our study could help to evaluate risk for UCEC patient and establishes personalized therapy.

\section{Conclusions}

In summary, the present study developed a standardized computational procedures to evaluate cancer patient hallmark-related risk based on genes and IncRNAs expression. All the UCEC patients were divided to diverse cancer hallmark-related risk groups and showed different prognosis. Some key cancer hallmark-related IncRNAs were significantly associated with UCEC development. Collectively, our study leads to a novel starting point for future functional explorations, the identification of biomarkers, and IncRNA-based targeted therapy for UCEC.

\section{Abbreviations}

UCEC: uterine corpus endometrioid carcinoma; IncRNA: long non-coding RNA; TCGA: The Cancer Genome Atlas; GO: gene ontology; PCC: Pearson correlation coefficients.

\section{Declarations}

\section{Funding}

Not applicable.

\section{Disclosure of interest}

The authors declare that they have no competing interest.

\section{Acknowledgments}

Not applicable.

\section{Availability of data and material}

The data that support the findings of this study are available from the corresponding author upon reasonable request.

\section{Ethics approval and consent to participate}

Not applicable.

\section{Patient consent for publication}


Not applicable.

Competing interests

The authors declare that they have no competing interests.

Consent for publication

Not applicable.

Authors' contributions

LN conceived and designed the experiments, ZSY, LY, LTY and CXD analysed the data, and LN and ZSY wrote the manuscript.

\section{References}

1. Bray F, Ferlay J, Soerjomataram I, Siegel RL, Torre LA, Jemal A: Global cancer statistics 2018: GLOBOCAN estimates of incidence and mortality worldwide for 36 cancers in 185 countries. $C A$ Cancer J Clin 2018, 68:394-424.

2. Gaber C, Meza R, Ruterbusch JJ, Cote ML: Endometrial Cancer Trends by Race and Histology in the USA: Projecting the Number of New Cases from 2015 to 2040. J Racial Ethn Health Disparities 2016.

3. Siegel RL, Miller KD, Jemal A: Cancer statistics, 2020. CA Cancer J Clin 2020, 70:7-30.

4. Bell DW, Ellenson LH: Molecular Genetics of Endometrial Carcinoma. Annu Rev Patho/ 2019, 14:339367.

5. Anastasiadou E, Jacob LS, Slack FJ: Non-coding RNA networks in cancer. Nat Rev Cancer 2018, 18:518.

6. Kaikkonen MU, Adelman K: Emerging Roles of Non-Coding RNA Transcription. Trends Biochem Sci 2018, 43:654-667.

7. Ransohoff JD, Wei Y, Khavari PA: The functions and unique features of long intergenic non-coding RNA. Nat Rev Mol Cell Biol 2018, 19:143-157.

8. Uszczynska-Ratajczak B, Lagarde J, Frankish A, Guigo R, Johnson R: Towards a complete map of the human long non-coding RNA transcriptome. Nat Rev Genet 2018, 19:535-548.

9. Wang Y, Fang Z, Hong M, Yang D, Xie W: Long-noncoding RNAs (IncRNAs) in drug metabolism and disposition, implications in cancer chemo-resistance. Acta Pharm Sin B 2020, 10:105-112.

10. Wu P, Mo Y, Peng M, Tang T, Zhong Y, Deng X, Xiong F, Guo C, Wu X, Li Y, et al: Emerging role of tumor-related functional peptides encoded by IncRNA and circRNA. Mol Cancer 2020, 19:22.

11. Zhang G, Ma A, Jin Y, Pan G, Wang C: LncRNA SNHG16 induced by TFAP2A modulates glycolysis and proliferation of endometrial carcinoma through miR-490-3p/HK2 axis. Am J Trans/ Res 2019, 11:7137-7145. 
12. Wang E, Zaman N, McGee S, Milanese JS, Masoudi-Nejad A, O'Connor-McCourt M: Predictive genomics: a cancer hallmark network framework for predicting tumor clinical phenotypes using genome sequencing data. Semin Cancer Biol 2015, 30:4-12.

13. Hanahan D, Weinberg RA: The hallmarks of cancer. Cell 2000, 100:57-70.

14. Hanahan D, Weinberg RA: Hallmarks of cancer: the next generation. Cel/ 2011, 144:646-674.

15. Plaisier CL, Pan M, Baliga NS: A miRNA-regulatory network explains how dysregulated miRNAs perturb oncogenic processes across diverse cancers. Genome Res 2012, 22:2302-2314.

16. Carbon S, Ireland A, Mungall CJ, Shu S, Marshall B, Lewis S, Ami GOH, Web Presence Working G: AmiGO: online access to ontology and annotation data. Bioinformatics 2009, 25:288-289.

17. Kuleshov MV, Jones MR, Rouillard AD, Fernandez NF, Duan Q, Wang Z, Koplev S, Jenkins SL, Jagodnik KM, Lachmann A, et al: Enrichr: a comprehensive gene set enrichment analysis web server 2016 update. Nucleic Acids Res 2016, 44:W90-97.

18. Sho T, Hachisuga T, Nguyen TT, Urabe R, Kurita T, Kagami S, Kawagoe T, Matsuura Y, Shimajiri S: Expression of estrogen receptor-alpha as a prognostic factor in patients with uterine serous carcinoma. Int J Gynecol Cancer 2014, 24:102-106.

19. Togami S, Sasajima Y, Oi T, Ishikawa M, Onda T, Ikeda S, Kato T, Tsuda H, Kasamatsu T: Clinicopathological and prognostic impact of human epidermal growth factor receptor type 2 (HER2) and hormone receptor expression in uterine papillary serous carcinoma. Cancer Sci 2012, 103:926932.

20. Nishimura M, Saito T, Yamasaki H, Kudo R: Suppression of gap junctional intercellular communication via 5' $\mathrm{CpG}$ island methylation in promoter region of E-cadherin gene in endometrial cancer cells. Carcinogenesis 2003, 24:1615-1623.

21. Wright GW, Huang DW, Phelan JD, Coulibaly ZA, Roulland S, Young RM, Wang JQ, Schmitz R, Morin RD, Tang J, et al: A Probabilistic Classification Tool for Genetic Subtypes of Diffuse Large B Cell Lymphoma with Therapeutic Implications. Cancer Cell 2020, 37:551-568 e514.

22. Lee S, Lim S, Lee T, Sung I, Kim S: Cancer subtype classification and modeling by pathway attention and propagation. Bioinformatics 2020.

\section{Figures}


(1). Identification of UCEC-specific gene and InCRNA co-expressed pairs based on expression profiles
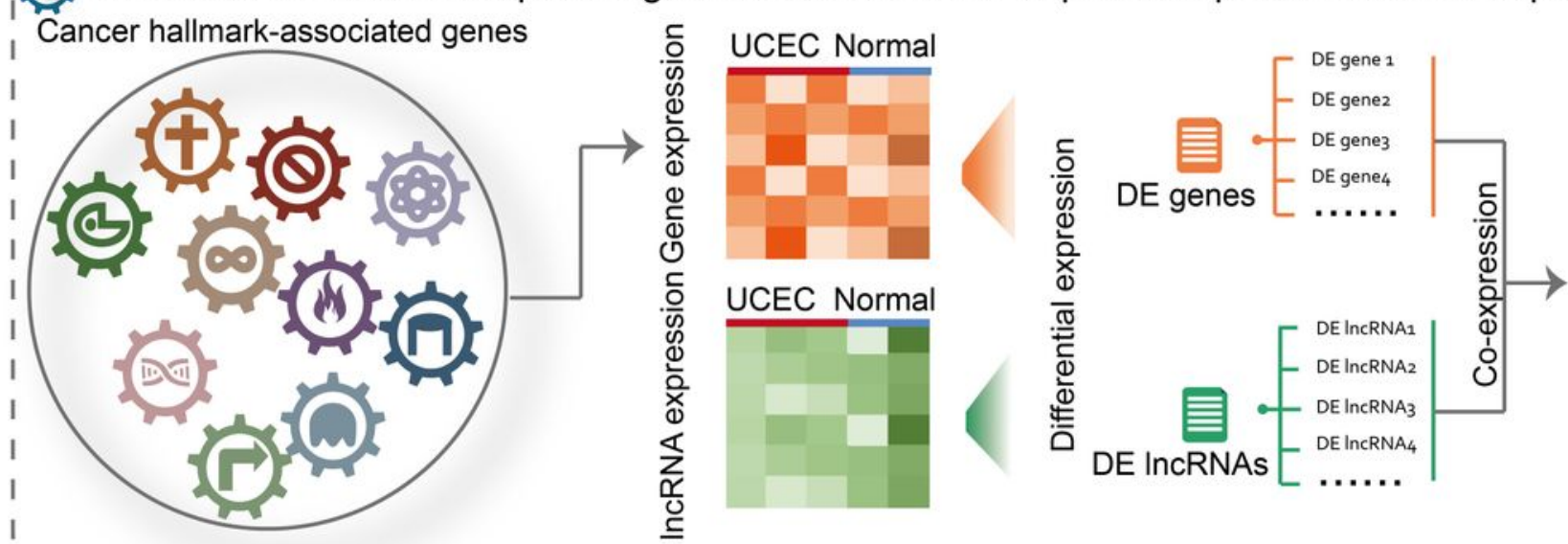

PCCs

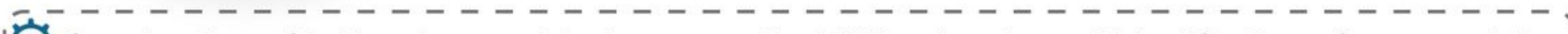

2. Construction of hallmark-associated gene and IncRNA networks and identification of core modules
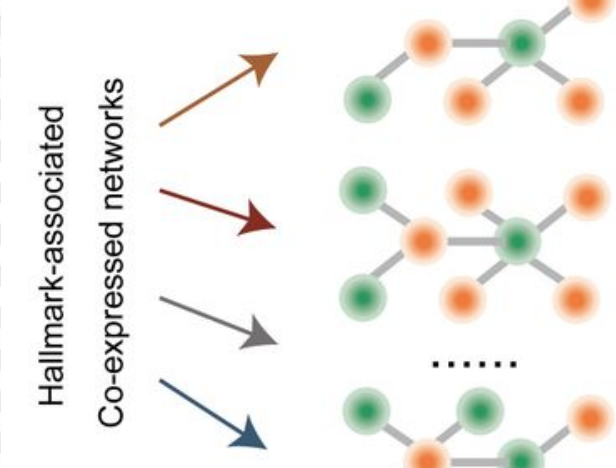

MCODE

Identify core modules

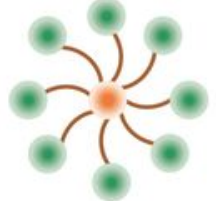

for each hallmark
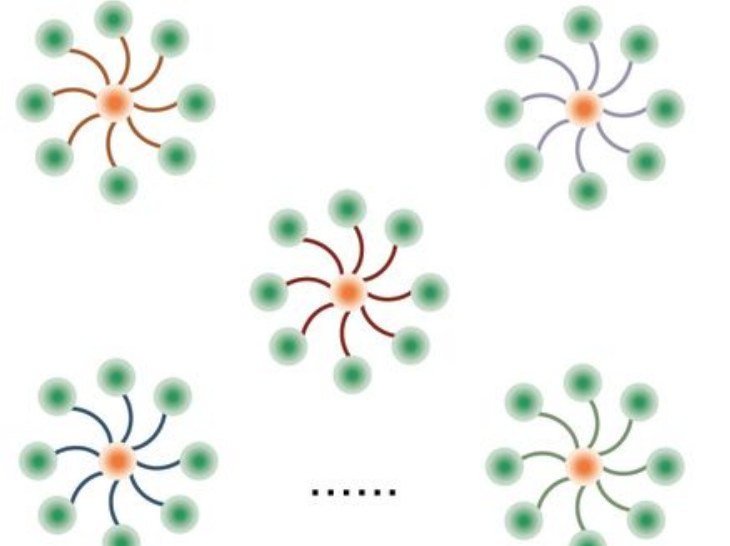

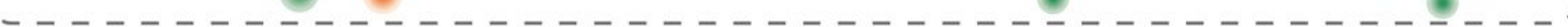

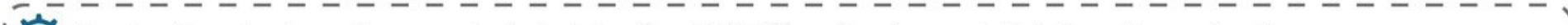

13. Evaluating hallmark-associated risks for UCEC patients and dividing them to diverse groups

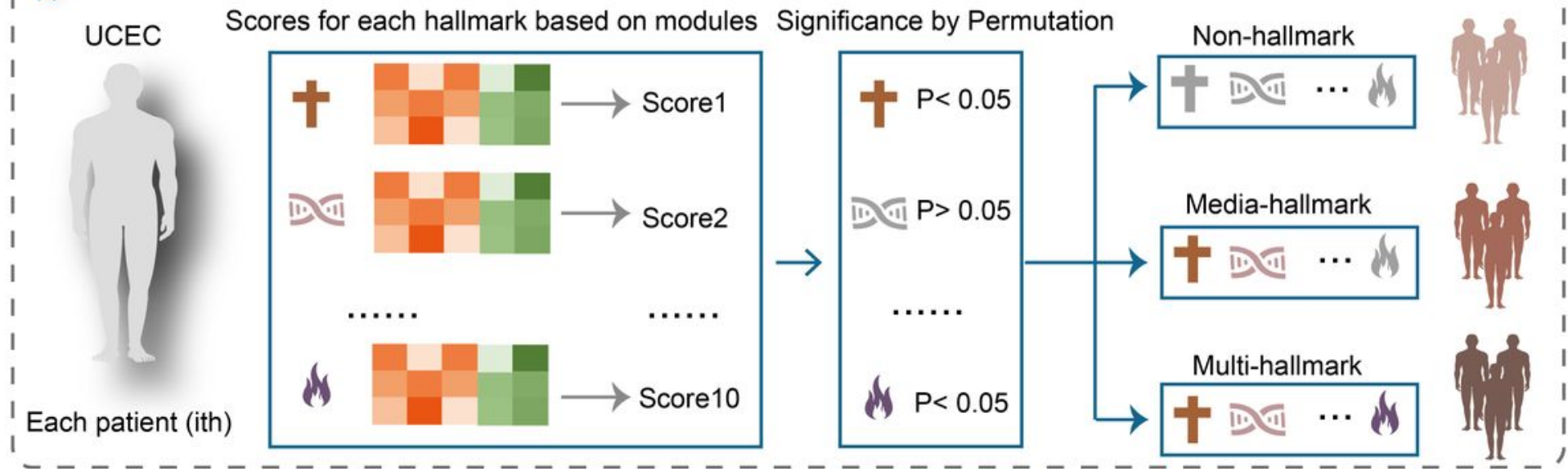

Figure 1

The workflow of evaluating cancer hallmark-related risk for UCEC patients based on genes and IncRNAs expression. Step 1. Identification of UCEC-specific cancer hallmark-related genes and IncRNAs and calculating co-expression pairs based on genes and IncRNAs expression profiles. Step 2. Construction of UCEC-specific cancer hallmark-related genes and IncRNAs co-expressed networks and extraction of core 
modules. Step 2. Evaluating cancer hallmark-related risks for UCEC patients and dividing them to diverse groups.

A
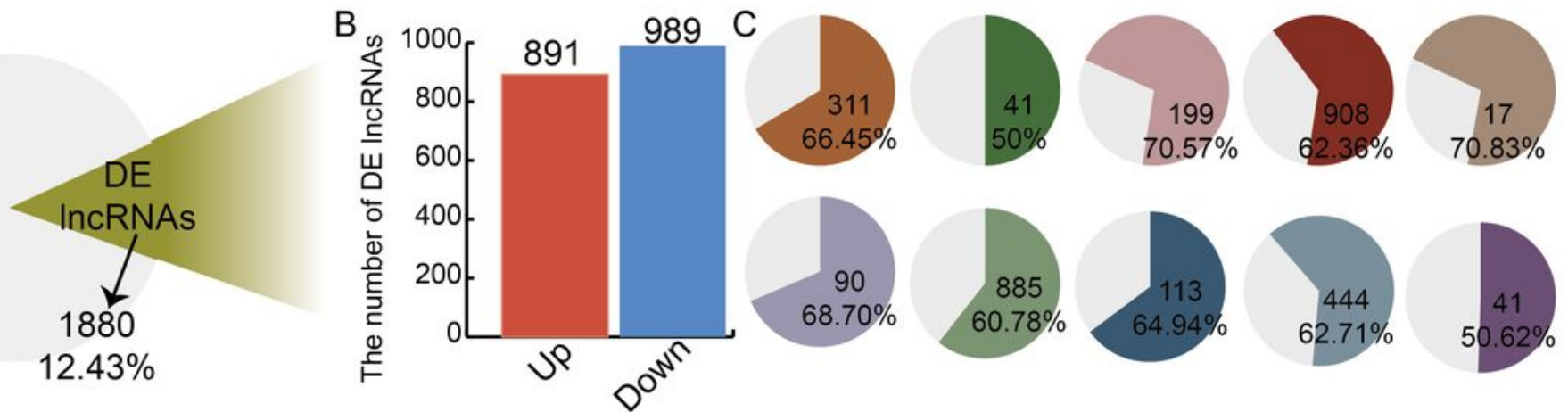

$12.43 \%$

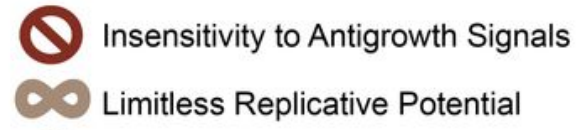
Reprogramming Energy Metabolism
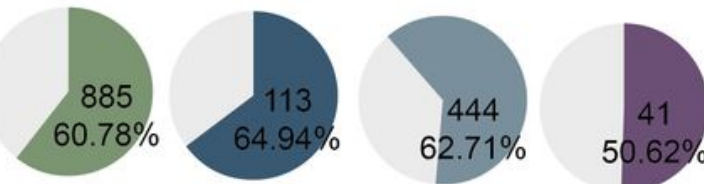

늘 Evading Apoptosis

6 Evading Immune Detection

ion
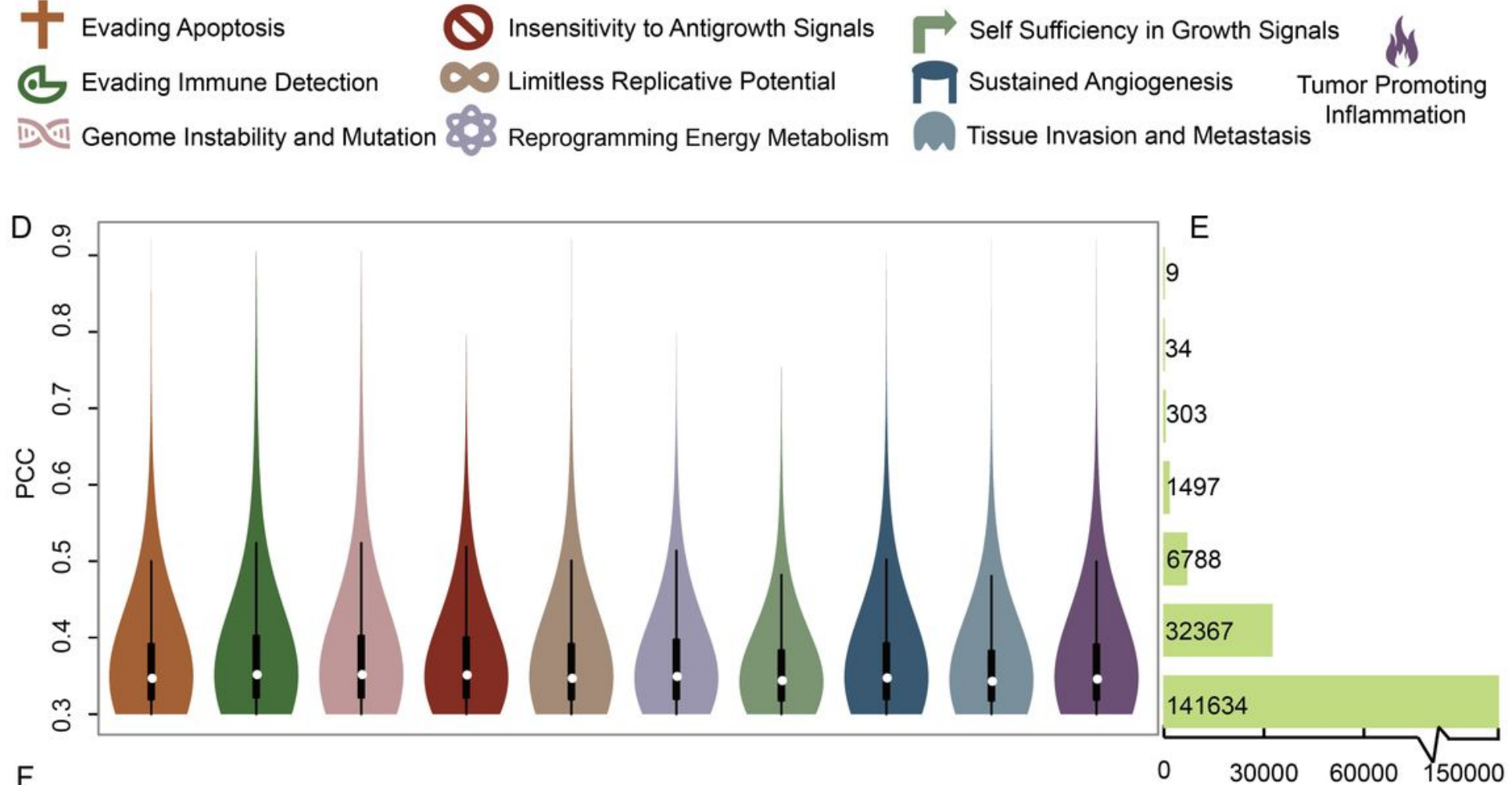

$\mathrm{F}$

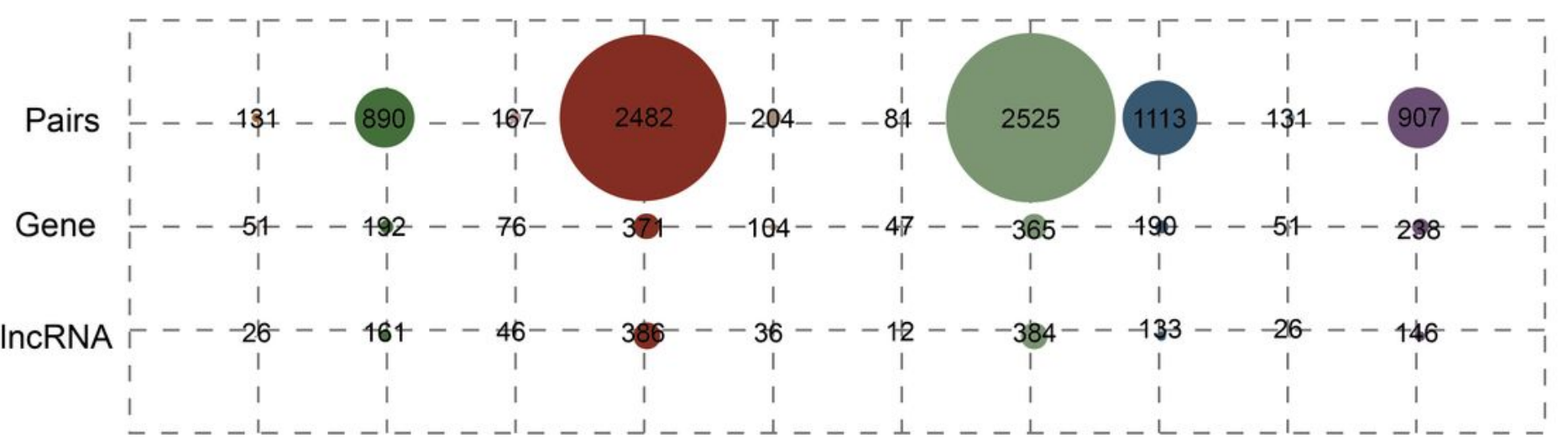

\section{Figure 2}

Some IncRNAs and cancer hallmark-related genes were specific and co-expressed in UCEC. (A) The pie chart shows percent of differential expressed IncRNAs in UCEC. (B) The bar plots show numbers of up(red) and down-regulated (blue) IncRNAs. (C) Pie charts show percents of differential expressed genes in 
UCEC for each kind of cancer hallmark. (D) The violin plots show PCCs between UCEC-specific IncRNAs and genes in each cancer hallmark. (E) The bar charts show numbers of co-expressed UCEC-specific IncRNAs and genes pairs. (F) The size of the circle represent the number of co-expressed UCEC-specific IncRNAs and genes pairs, genes and IncRNAs in each cancer hallmark.
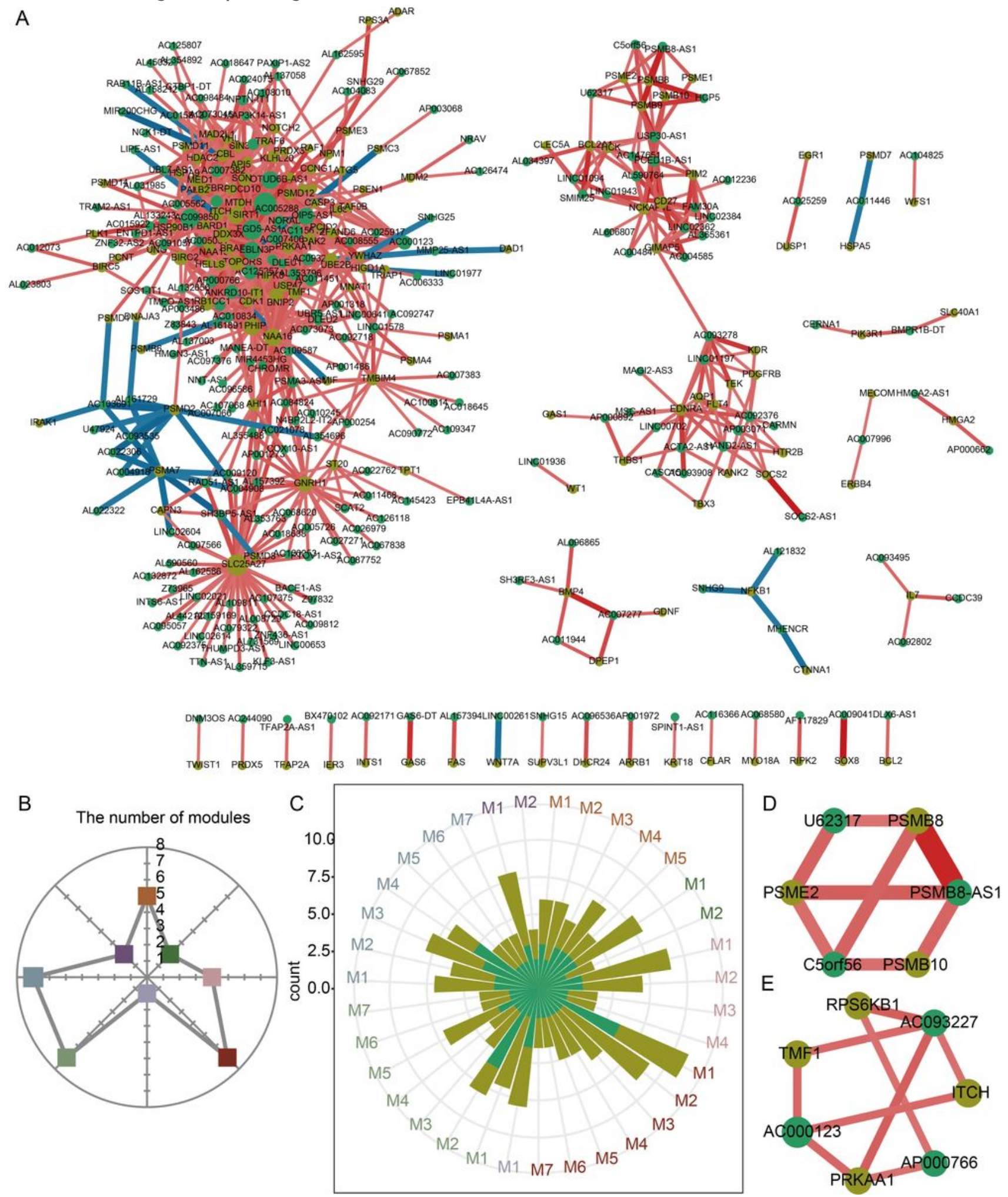

Figure 3 
Extraction of core modules from co-expressed IncRNAs and genes networks in each cancer hallmark. (A) Co-expressed UCEC-specific IncRNAs and genes networks in cancer hallmark evading apoptosis. Green and yellow nodes represent IncRNAs and genes, respectively. (B) The radar chart shows numbers of core modules in each cancer hallmark. (C) The rose diagram shows numbers of IncRNAs and genes in each core module. (D, E) Two core modules as examples in cancer hallmark evading apoptosis.
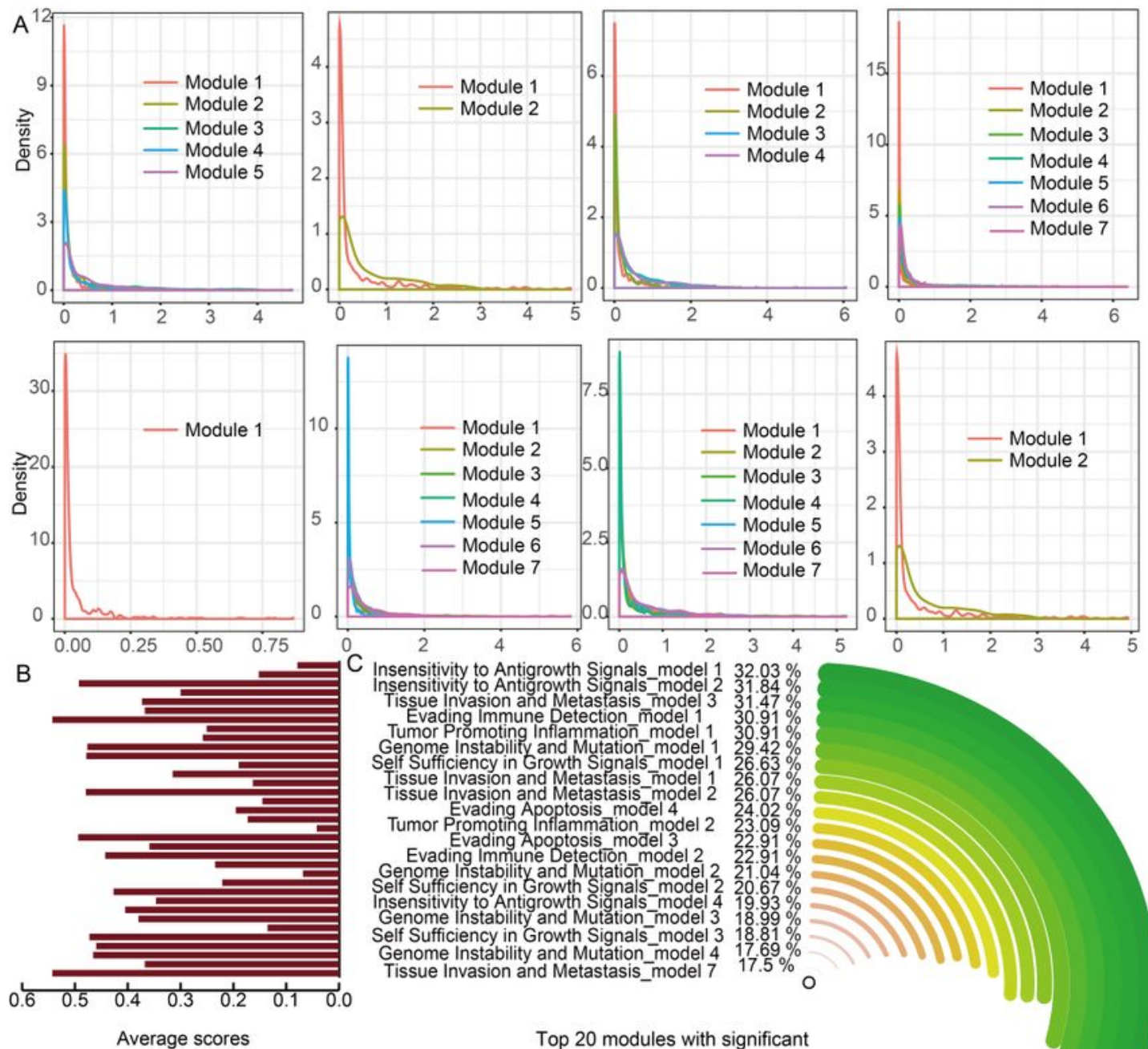

Average scores

Top 20 modules with significant hallmark-associated samples

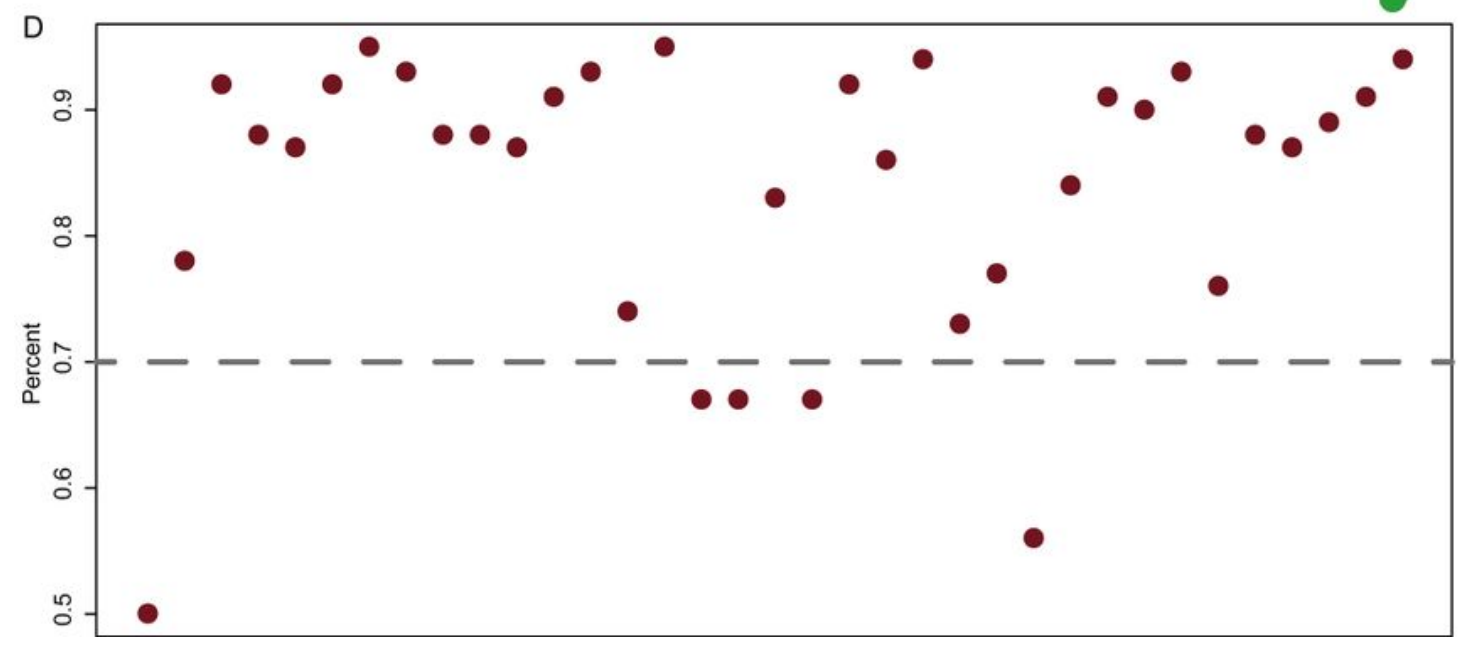

Figure 4 
Evaluating specific cancer hallmark-related risk for each UCEC patient based on core modules. (A) Density curves of risk scores for all core modules in each cancer hallmark. (B) The bar charts show average risk scores for top 20 core modules with significant cancer hallmark-related UCEC samples. (C) The diagram shows percents of significant risk UCEC samples using diverse core modules. (D) The point plot shows percents of UCEC samples ranked before corresponding orders in all core modules.

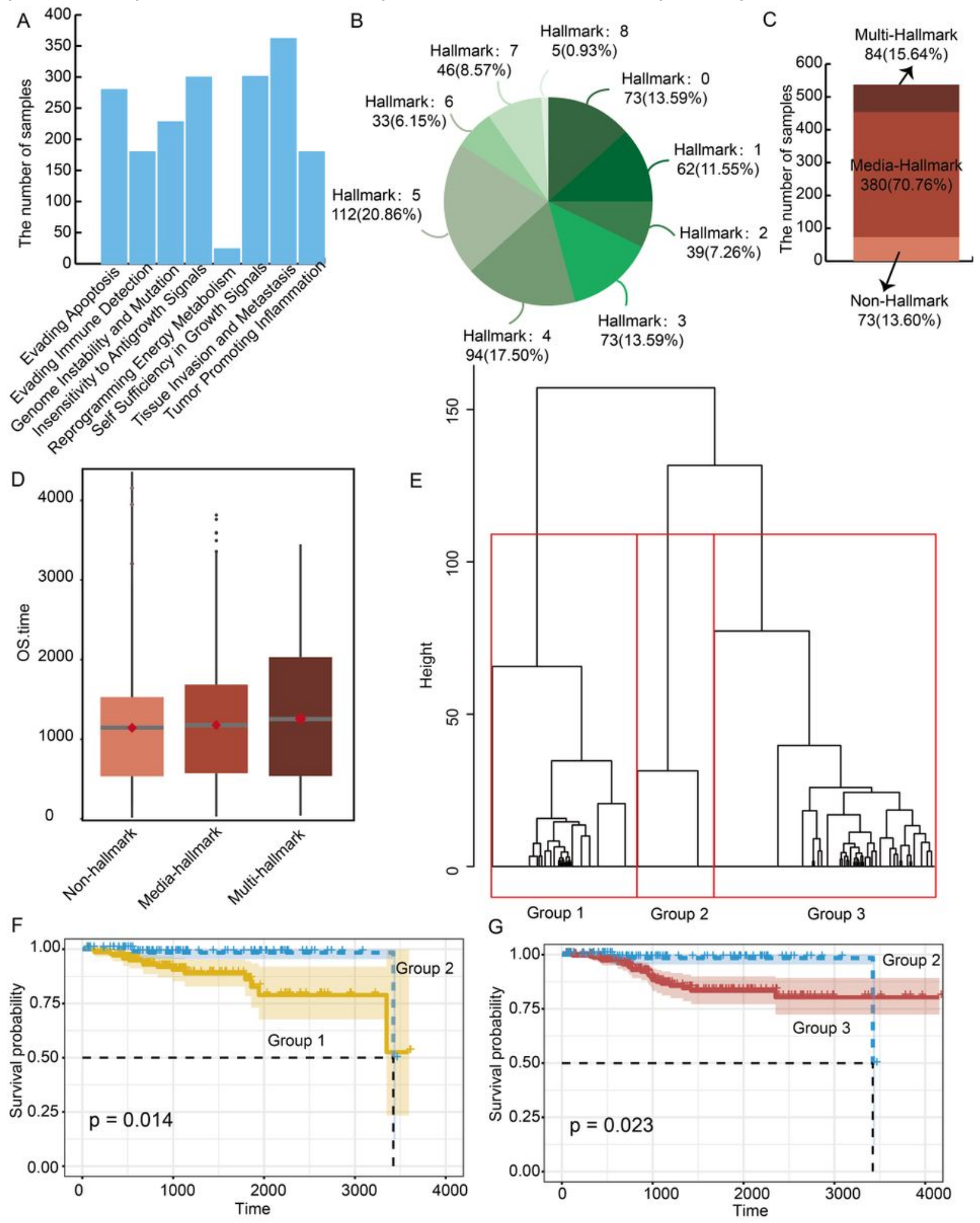

Figure 5 
Specific features of UCEC groups with diverse cancer hallmark risk. (A) The bar plot shows numbers of samples related to diverse cancer hallmark. (B) The pie chart shows percents of UCEC samples with different numbers of cancer hallmarks. (C) The numbers of UCEC samples in diverse cancer hallmarkrelated risk groups. (D) The box plots show overall survival days in diverse cancer hallmark-related risk groups. (E) Clustering trees for UCEC samples. (F, G) The Kaplan-Meier curve for the overall survival of two cancer hallmark-related risk groups. The difference between the two curves was evaluated by a twosided log-rank test.
A

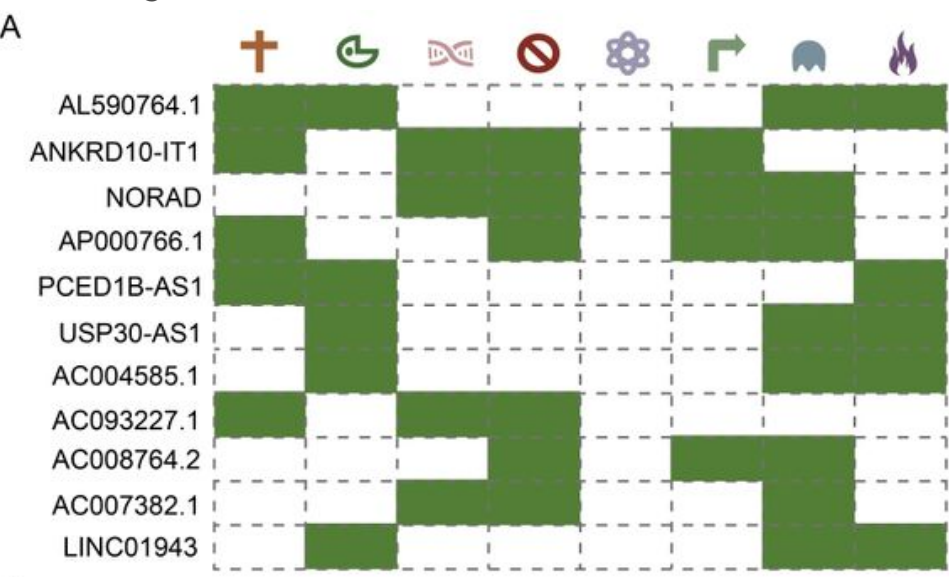
B
C gap junction assembly
negative regulation of intracellular estrogen receptor signaling negative regulation of intracellular steroid hormone receptor signaling regulation of intracellular estrogen receptor signaling pathway exonucleolytic nuclear-transcribed mRNA catabolic process involved nuclear-transcribed mRNA catabolic process, exonucleolytic huclear-transcribed mRNA catabolic process, deadepylatiop-dependent, decay,
exonucleolytic nuclear-transcribed mRNA catabolic process
nuclear-transcribed mRNA catabolic process, exonucleolytic huclear-transcribed mRNA catabolic process, deadenylation-dependent decay
regulation of Wnt signaling pathway
\begin{tabular}{lllll}
\hline 0.0 & 0.5 & 1.0 & 1.5 & 2.0
\end{tabular}
egulation of cellular macromolecule biosynthetic process regulation of cellular amide metabolic process posttranscriptional regulation of gene expression regulation of cellular protein metabolic process positive regulation of cellular amide metabolic process positive regulation of translation
positive regulation of cellular protein metabolic process regulation of kinase activity
huclear-transcribed mRNA catabolic process,
honsense-mediated decay
positivę regulation of macromolecule biosynthetic process $\begin{array}{lllllll}0.0 & 0.5 & 1.0 & 1.5 & 2.0 & 2.5\end{array}$

gap junction assembly
protein $\mathrm{K} 11$-linked deubiquitination
mitochondrial fusion
organelle fusion
mitochondrion disassembly
regulation of cellular amide metabolic process
autophagy of mitochondrion
posttranscriptional regulation of gene expression
regulation of cellular protein metabolic process
membrane fusion
$0.0 \quad 0.5 \quad 1.0 \quad 1.5 \quad 2.0 \quad 2.5$

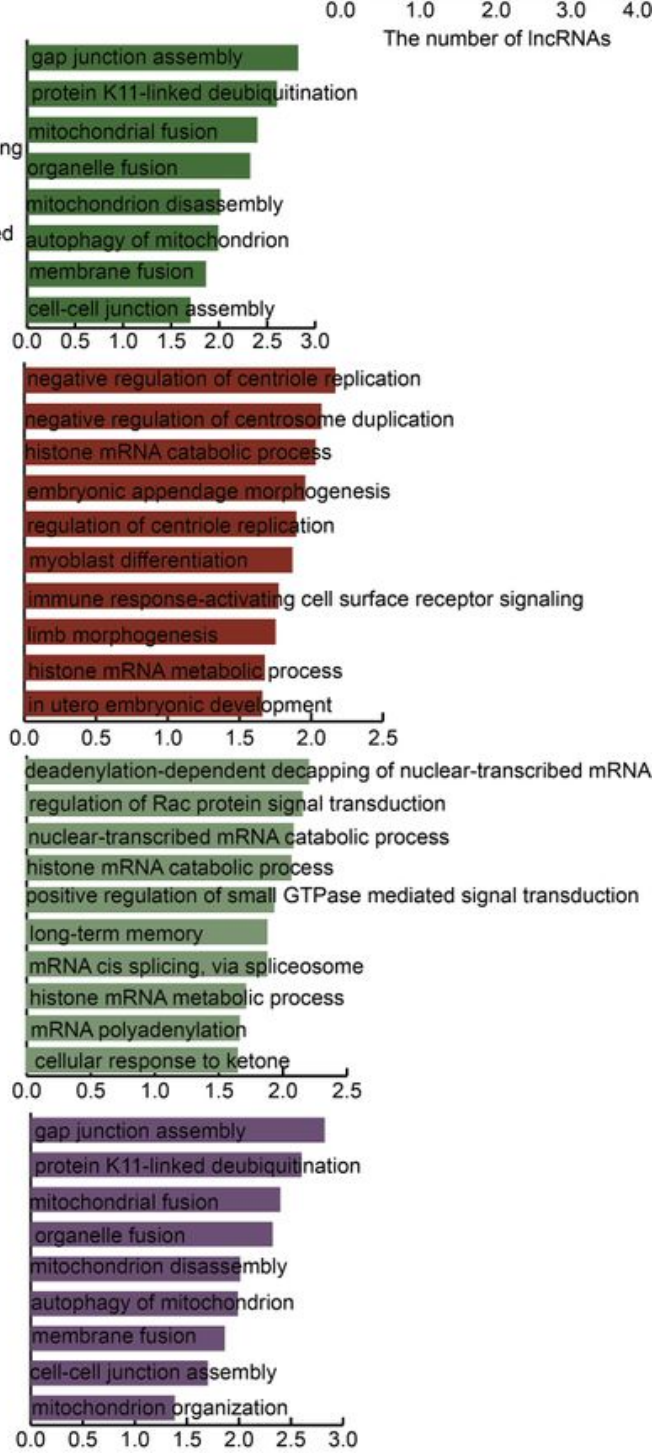


Figure 6

Some key IncRNAs could participate in multiple kinds of cancer hallmarks and showed specific functions.

(A) The heatmap shows IncRNAs if are associated with cancer hallmark. (B) The bar plots show numbers of cance hallmarks which IncRNAs participate in. (C) Enrichment pathways of IncRNAs in each cancer hallmark. The bar plots represent -log (P). 